\title{
EVALUACIÓN DE LA ESTANCIA HOSPITALARIA EN PACIENTES COLECISTECTOMIZADOS EN EL HOSPITAL NACIONAL DOS DE MAYO, LIMA-PERÚ
}

\author{
EVALUATION OF HOSPITAL STAY IN COLECISTECTOMIZED PATIENTS IN THE \\ HOSPITAL NACIONAL DOS DE MAYO, LIMA-PERU
}

Zoila Alejandra Villar-Manosalva1,a, Magdiel José Manuel Gonzales-Menéndez²,a,b,c, Cecilia Roxana Salinas-Salas ${ }^{1, a, d, e .}$

\begin{abstract}
RESUMEN
Objetivos: Evaluar la estancia hospitalaria en pacientes colecistectomizados por vía laparoscópica del sexo masculino en relación al sexo femenino. Métodos: Estudio observacional, retrospectivo, transversal. Se revisaron 514 historias de pacientes sometidos a colecistectomía laparoscópica en el Hospital nacional Dos de mayo julio del 2014 a julio de 2015. Resultados: Se estudiaron 514 pacientes, 374 (73\%) de sexo femenino y 140 (27\%) de sexo masculino (2.7:1); el grupo etáreo predominante fue de 47-62 años para ambos sexos y 45 años la edad promedio para el sexo femenino y 49 años para el masculino. La estancia hospitalaria promedio para ambos sexos fue de 2.66 días, 2.18 días para mujeres y 3.95 días para varones. El tiempo operatorio promedio fue de 86.97 minutos, 103.43 minutos en el sexo masculino y 80.80 minutos en el femenino. La complicación más frecuente fue la disección compleja, 4.5\% en el sexo masculino y $3.7 \%$ en el femenino; 1 de cada 13 mujeres y 1 de cada 5 hombres presentaron alguna complicación postoperatoria. El diagnóstico post operatorio más frecuente fue colecistitis crónica litiásica (79.2\%), le sigue la colecistitis subaguda (6\%), colecistitis aguda (5.6\%), piocolecisto (2.5\%), Pólipo vesicular (1.9\%), hidrocolecisto (9\%), plastrón vesicular (5\%), Hidropiocolecisto (5\%) y síndrome de Mirizzi (5\%). Conclusión: La estancia hospitalaria es mayor en el sexo masculino, así mismo tienen mayor tiempo operatorio por mayor dificultad en la disección y diagnósticos más complicados.
\end{abstract}

Palabras clave: Sexo masculino; Estancia hospitalaria; Colecistectomía laparoscópica. (fuente: DeCS BIREME)

\begin{abstract}
Objective: Evaluate the hospital stay in laparoscopic cholecystectomy patients of the male sex in relation to the female sex. Methods: Observational, retrospective, cross-sectional study. 514 records of patients undergoing laparoscopic cholecystectomy in the "Hospital nacional Dos de mayo" in the one-year period were reviewed. Results: Of the 514 patients $374(73 \%)$ were female and $140(27 \%)$ males with a ratio of 2.7: 1, the predominant age group was 47-62 years for both sexes, and the average age for females was 45 years and 49 years for men. The average hospital stay for both sexes was 2.66 days, 2.18 days to 3.95 days for women and men. The average operating time was 86.97 minutes, 103.43 minutes in males and 80.80 minutes in the women. The most common complication was complex dissection, 4.5\% in males and 3.7\% in women. 1 in 13 women and 1 in 5 men developed a postoperative complication. The most common postoperative diagnosis was chronic cholelithiasis (79.2\%), followed by sub-acute cholecystitis (6\%), acute cholecystitis (5.6\%), empyema (2.5\%), gallbladder polyp (1.9\%), hydrocholecystis (9\%), vesicular plastron (5\%), cholecystitis with empyema (5\%) and Mirizzi syndrome (5\%). Conclusions: In the present study we found that hospitalization is higher in males; also they have longer operating time for dissection more difficult and more complicated diagnoses.
\end{abstract}

Key words: Male gender; Hospital stay; Laparoscopic cholecystectomy. (source: MeSH NLM)

\footnotetext{
Internado Médico del Hospital Nacional Dos de Mayo, Lima-Perú.

${ }^{2}$ Instituto de Investigación en Ciencias Biomédicas, Facultad de Medicina Humana, Universidad Ricardo Palma, Lima, Perú.

aédico cirujano, b Maestro en Medicina con Mención en Cirugía General, ' Especialista en Cirugía General,

'Magister en Medicina, e Especialista en Geriatría.
}

Correspondencia: Zoila Alejandra Villar Manosalva. Dirección: Jr. Bodegones 591 Las Gardenias, Surco; Lima, Perú. Teléfono: 2560860. Celular: 992844230. Correo: aleja_dsk@hotmail.com

Citar como: Zoila Alejandra Villar-Manosalva, Magdiel José Manuel Gonzales-Menéndez, Cecilia Roxana Salinas-Salas: Evaluación de la estancia hospitalaria en pacientes colecistectomizados en el Hospital Nacional Dos de Mayo, lima-Perú. [Artículo original]. Rev. Fac. Med. Hum. $2017 ; 17(1): 57-63$. DOI 10.25176/RFMH.v17.n1.749 


\section{INTRODUCCIÓN}

En Perú en 1990 se iniciaron las operaciones de colecistectomía laparoscópica y rápidamente se ha convertido en el tratamiento de elección de la colelitiasis por sus ventajas como: menor tiempo de hospitalización, menor dolor post operatorio y rápido reintegro laboral, en comparación a la colecistectomía clásica. Hoy en día, sus resultados se comparan favorablemente con la técnica clásica abierta.

Los factores de riesgo en la colecistectomía han sido bien tipificados y tienen relación con la edad, cirugía urgencia, presencia de cirugía abdominal previa, obesidad y enfermedades concomitantes.

La severidad del cuadro agudo desencadena principalmente una mayor dificultad técnica que incrementa las lesiones transoperatorias, lo que le da una importancia mayor a los pacientes del género masculino.

Los factores etiológicos de la relación género masculino con aumento de la mortalidad podría estar relacionado específicamente con dos situaciones: la primera es que generalmente el paciente masculino tiene más edad cuando se somete a la cirugía y, por otro lado, es sometido a cirugía urgente con mayor frecuencia, la explicación podría estar fundada en que las pacientes del género femenino son más sensibles al proceso inflamatorio y clínicamente es más florida la sintomatología, provocando que el tratamiento quirúrgico se realice mucho más rápido. Es entonces que el género como factor de conversión a cirugía abierta ha atraído atención en los últimos años, y muchos estudios han demostrado que los cálculos biliares sintomáticos, la inflamación y la fibrosis son más extensos en los hombres que en las mujeres. Los hallazgos apoyan la observación de una mayor tasa de conversión en hombres que en mujeres' ${ }^{1}$. No se ha podido llegar a demostrar que el género masculino es un factor de riesgo para la conversión; en su lugar, describen una combinación de factores de riesgo, incluyendo la edad avanzada, el modo de presentación patológica (colecistitis aguda), y una historia de cirugía abdominal superior ${ }^{2,3}$.

En el hospital Dos de mayo, el empleo de la técnica laparoscópica durante los últimos años ha permitido reunir una casuística significativa, cuyas características demográficas y resultados quirúrgicos al ser analizados permiten reconocer instancias en las cuales todavía se puede optimizar al máximo los resultados a través de modificaciones del manejo peri e intraoperatorio.

En la investigación que presentamos se evaluó la colecistectomía laparoscópica en el varón en relación a una técnica más complicada y mayor estancia hospitalaria en comparación con que en el género femenino.

\section{MÉTODOS}

Se realizó un estudio no experimental, retrospectivo. Durante el período julio del 2014 a julio del 2015, en el Hospital Nacional Dos de Mayo, se encontraron 598 pacientes sometidos a colecistectomía laparoscópica electiva de los cuáles 514 pacientes cumplían con los criterios de inclusión y exclusión.

\section{Criterios de inclusión:}

- Pacientes mayores de 15 años de ambos sexos, colecistectomizados por vía laparoscópica hospitalizados en los servicios de Cirugía del Hospital Dos de Mayo de mayo de julio del 2014 a julio del 2015.

\section{Criterios de exclusión:}

- Pacientes que tengan además el diagnóstico de enfermedad hepática o cáncer de vesícula biliar.

- Pacientes mayores de 75 años.

- Pacientes cuyas historias estén incompletas.

Se tomaron los registros estadísticos de los procedimientos quirúrgicos llevados a cabo por los Servicios de Cirugía General desde Julio de 2014 a Julio del 2015, las historias clínicos de los pacientes a los que se les realizó Colecistectomía electiva durante este periodo de tiempo. Identificándose a los pacientes que presentaron complicaciones durante la Colecistectomía o después de ella, de acuerdo a los dictados quirúrgicos y a los reportes operatorios, registrando sus datos en una tabla de datos elaborada con el programa de cómputo Microsoft Office Excel 2010.

El análisis de las variables se ejecutó con el programa estadístico SPSS versión 17. Se evaluó la relación entre las variables de exposición (varón o mujer) y las de resultado (estancia hospitalaria, eventos intraoperatorios inesperados, conversión y tiempo operatorio) mediante el análisis bivariado. Además de ello se usó la prueba de $x^{2}$ para evaluar asociación entre variables categóricas (por ejemplo, entre sexo y conversión operatoria) y la prueba de $t$ de student para datos no apareados la empleamos para la comparación de medias. Se consideró significativas las $p<0,05$ para comparar variables continuas entre dos grupos (por ejemplo, tiempo operatorio entre ambos grupos de sexo) y dependiendo del comportamiento (distribución normal o no) de la variable en cuestión. 


\section{RESULTADOS}

Tabla 1. Características generales de los pacientes según género.

\begin{tabular}{lccc|}
\hline & Femenino & Masculino & Total \\
\hline Sexo & $374(72.8 \%)$ & $40(27.2 \%)$ & $514(100 \%)$ \\
\hline Edad (años) & $44(44.36)$ & $49(48.72)$ & $45.54(15-75)$ \\
\hline Tiempo operatorio (promedio) & $80.80(25-220)$ & $103.43(30-480)$ & $86.97(25-480)$ \\
\hline Estancia post operatoria (promedio-días) & $2.18(1-12)$ & $3.95(1-29)$ & $2.66(1-29)$ \\
\hline
\end{tabular}

Fuente: INICIB-FAMURP/ HOSPITAL DOS DE MAYO

De los 514 pacientes, 374 fueron de sexo femenino y 140 de sexo masculino; la edad promedio fue de 45 años, 44 años en promedio para mujeres y 49 años en promedio para varones. El tiempo operatorio promedio para ambos fue de 86.97 minutos, 80.8 minutos para el sexo femenino y 103.43 minutos para el sexo masculino. La estancia hospitalaria promedio para todos los pacientes fue de 2.66 días, 2.18 días para mujeres y 3.95 días para varones.

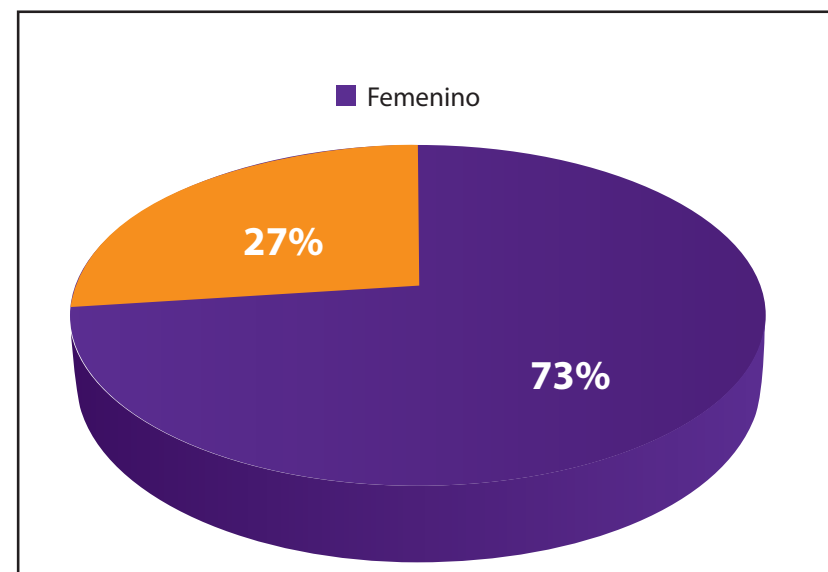

Fuente: INICIB-FAMURP/ HOSPITAL DOS DE MAYO

Gráfico 1. Distribución por género.

El género que predominó fue el femenino siendo un $73 \%$ (374 pacientes) sobre el masculino con $27 \%$ (140 pacientes).

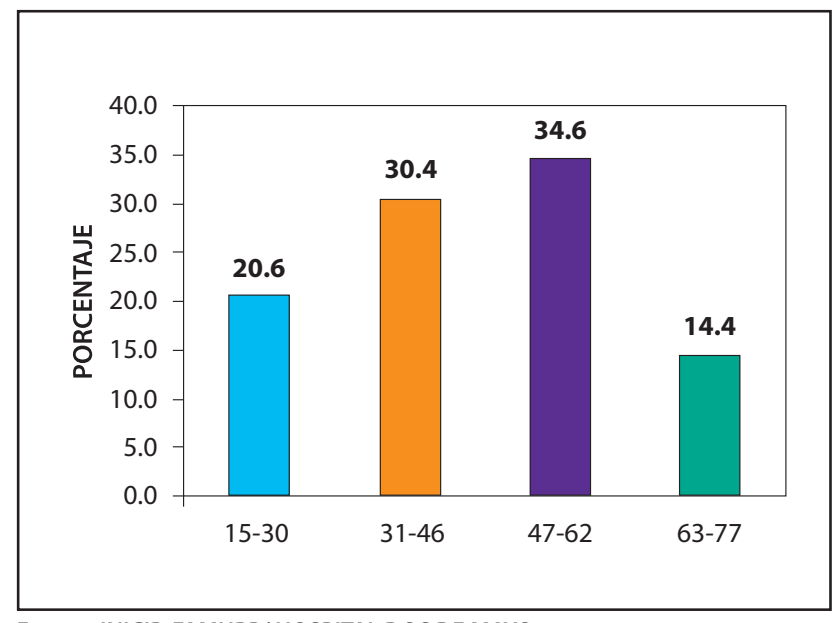

Fuente: INICIB-FAMURP/ HOSPITAL DOS DE MAYO

Gráfico 2. Edad categorizada por gru pos etáreos quinquenales.

El grupo etáreo predomínate fue de 47-62 años con 34.6\%.

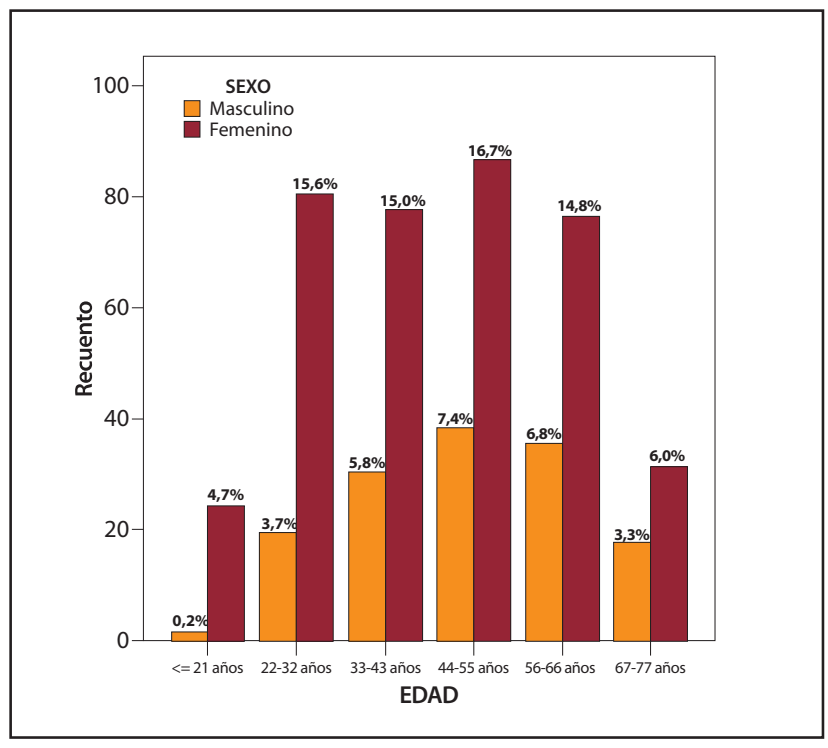

Fuente: INICIB-FAMURP/ HOSPITAL DOS DE MAYO

Gráfico 3. Distribución según género y grupo etáreos.

En ambos sexos predomino el grupo etáreo de $44-55$ años.

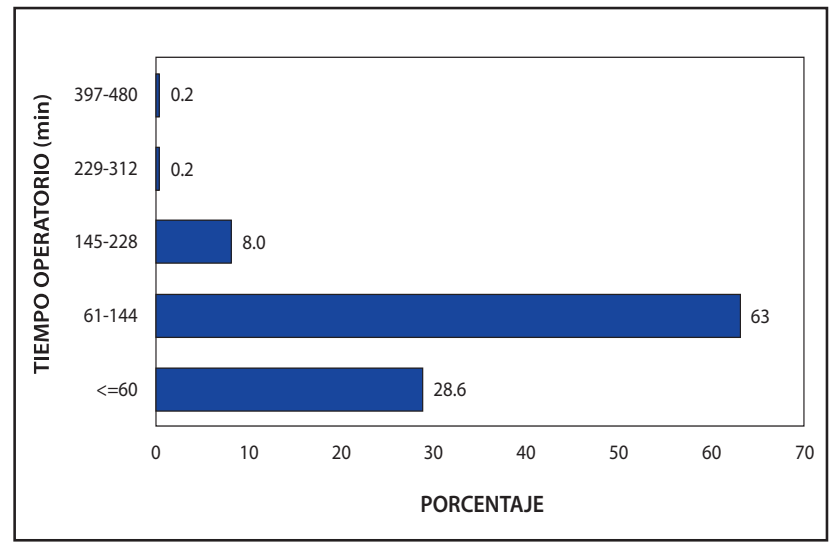

Fuente: INICIB-FAMURP/ HOSPITAL DOS DE MAYO

Gráfico 4. Tiempo operatorio de los pacientes sometidos a Colecistectomía Laparoscópica.

\begin{tabular}{ll}
\hline Promedio & $86.97 \mathrm{~min}(25-480 \mathrm{~min})$ \\
Desviación típica & 40,062 \\
$\mathrm{p}$ &, 001 \\
\hline
\end{tabular}

El tiempo operatorio en promedio fue de 86.97 minutos, 324 pacientes (63\%) se encontraron dentro del rango de 61-144 minutos, 147 (28.6\%) con un tiempo menor de 60 minutos, 41 (8\%) entre 145-228 minutos, 1 paciente (0.2\%) entre 229312 minutos y $1(0.2 \%)$ entre $397-480$. 
Tabla 2. Distribución según género en cuanto a Complicaciones Intraoperatorias.

\begin{tabular}{|c|c|c|c|c|c|c|}
\hline \multirow[t]{3}{*}{$\begin{array}{l}\text { Complicación } \\
\text { Intraoperatoria }\end{array}$} & \multicolumn{4}{|c|}{ Sexo } & \multirow{2}{*}{\multicolumn{2}{|c|}{ Total }} \\
\hline & \multicolumn{2}{|c|}{ Femenino } & \multicolumn{2}{|c|}{ Masculino } & & \\
\hline & $\mathbf{N}^{\circ}$ & $\%$ & $\mathbf{N}^{\circ}$ & $\%$ & $\mathbf{N}^{\circ}$ & $\%$ \\
\hline Biliragia & 0 & $0.0 \%$ & 3 & $0.6 \%$ & 3 & $0.6 \%$ \\
\hline Ninguna & 347 & $67.5 \%$ & 89 & $17.3 \%$ & 436 & $84.8 \%$ \\
\hline Perforación vesicular & 1 & $0.2 \%$ & 2 & $0.4 \%$ & 3 & $0.6 \%$ \\
\hline Lesión del cístico & 1 & $0.2 \%$ & 0 & $0.0 \%$ & 1 & $0.2 \%$ \\
\hline Lesión del colédoco & 0 & $0.0 \%$ & 2 & $0.4 \%$ & 2 & $0.4 \%$ \\
\hline Hemorragia & 2 & $0.4 \%$ & 4 & $0.8 \%$ & 6 & $1.2 \%$ \\
\hline Disección Compleja & 19 & $3.7 \%$ & 23 & $4.5 \%$ & 42 & $8.2 \%$ \\
\hline Lesión Hepática & 0 & $0.0 \%$ & 1 & $0.2 \%$ & 1 & $0.2 \%$ \\
\hline Conversión & 4 & $0.8 \%$ & 16 & $3.1 \%$ & 20 & $3.9 \%$ \\
\hline Total & 374 & $72.8 \%$ & 140 & $27.2 \%$ & 514 & $100.0 \%$ \\
\hline
\end{tabular}

Fuente: INICIB-FAMURP/ HOSPITAL DOS DE MAYO

La complicación presentada en el intraoperatorio más frecuente fue la disección compleja de los elementos anatómicos con 42 pacientes (8.2\%) siendo19 del sexo femenino (3.7\%) y 23 del sexo masculino (4.5\%). El 84.8\% de los pacientes no presentó ninguna complicación.

Tabla 3. Distribución según género en cuanto a Complicaciones Postoperatorias.

\begin{tabular}{|c|c|c|c|c|c|c|}
\hline \multirow[t]{2}{*}{$\begin{array}{l}\text { Complicación } \\
\text { Postquirúrgicas }\end{array}$} & \multicolumn{4}{|c|}{ Sexo } & \multicolumn{2}{|c|}{ Total } \\
\hline & $\mathbf{N}^{\circ}$ & $\%$ & $\mathbf{N}^{\circ}$ & $\%$ & $\mathbf{N}^{\circ}$ & $\%$ \\
\hline Grado I & 18 & $3.5 \%$ & 16 & $3.1 \%$ & 34 & $6.6 \%$ \\
\hline Grado II & 7 & $1.4 \%$ & 16 & $3.1 \%$ & 23 & $4.5 \%$ \\
\hline Grado IIla & 0 & $0 \%$ & 1 & $0.2 \%$ & 1 & $0.2 \%$ \\
\hline Grado IIIb & 3 & $0.6 \%$ & 2 & $0.4 \%$ & 5 & $1.0 \%$ \\
\hline Grado IVa & 0 & $0.0 \%$ & 2 & $0.4 \%$ & 2 & $0.4 \%$ \\
\hline Ninguna & 346 & $67.3 \%$ & 103 & $20.0 \%$ & 449 & $87.3 \%$ \\
\hline Total & 374 & $72.8 \%$ & 140 & $27.2 \%$ & 514 & $100.0 \%$ \\
\hline
\end{tabular}

Fuente: INICIB-FAMURP/ HOSPITAL DOS DE MAYO

El 87\% de los pacientes no presentó ninguna complicación dentro del post operatorio siendo 346 mujeres (67.3\%) y 103 varones (20\%); 28 de 374 ( 1 de cada 13) mujeres tuvieron alguna complicación y 27 de 140 (1 de cada 5) varones tuvo alguna complicación. Dentro de las complicaciones la mayoría fue grado I con 34 pacientes (6.6\%) de los cuales 18 fueron mujeres (3.5\%) y 16 varones (3.1\%). Ningún paciente presentó disfunción multiorgánica (Grado IVb) ni falleció a causa de la cirugía (Grado V). 
Tabla 4. Distribución según género en cuanto a Diagnóstico post operatorio.

\begin{tabular}{|c|c|c|c|c|c|c|}
\hline \multirow[t]{3}{*}{$\begin{array}{c}\text { Diagnostico } \\
\text { Post Operatorio }\end{array}$} & \multicolumn{4}{|c|}{ Sexo } & \multirow{2}{*}{\multicolumn{2}{|c|}{ Total }} \\
\hline & \multicolumn{2}{|c|}{ Femenino } & \multicolumn{2}{|c|}{ Masculino } & & \\
\hline & $\mathbf{N}^{\circ}$ & $\%$ & $\mathbf{N}^{\circ}$ & $\%$ & $\mathbf{N}^{\circ}$ & $\%$ \\
\hline $\begin{array}{l}\text { Colecistitis Crónica } \\
\text { Litiásica }\end{array}$ & 314 & $61.1 \%$ & 93 & $18.1 \%$ & 407 & $79.2 \%$ \\
\hline Plastrón vesicular & 1 & $0.2 \%$ & 4 & $0.8 \%$ & 5 & $1.0 \%$ \\
\hline Colecistitis Aguda & 18 & $3.5 \%$ & 11 & $2.1 \%$ & 29 & $5.6 \%$ \\
\hline Hidrocolecisto & 7 & $1.4 \%$ & 2 & $.4 \%$ & 9 & $1.8 \%$ \\
\hline Piocolecisto & 6 & $1.2 \%$ & 7 & $1.4 \%$ & 13 & $2.5 \%$ \\
\hline Pólipo vesicular & 5 & $1.0 \%$ & 5 & $1.0 \%$ & 10 & $1.9 \%$ \\
\hline Hidropiocolecisto & 2 & $0.4 \%$ & 3 & $0.6 \%$ & 5 & $1.0 \%$ \\
\hline Síndrome de Mirizzi & 3 & $0.6 \%$ & 2 & $0.4 \%$ & 5 & $1.0 \%$ \\
\hline Colcistitis Subaguda & 18 & $3.5 \%$ & 13 & $2.5 \%$ & 31 & $6.0 \%$ \\
\hline Total & 374 & $72.8 \%$ & 140 & $27.2 \%$ & 514 & $100.0 \%$ \\
\hline
\end{tabular}

Fuente: INICIB-FAMURP/ HOSPITAL DOS DE MAYO

El diagnóstico post operatorio más frecuente fue de colecistitis crónica litiásica con 407 pacientes (79.2\%) de los cuáles 314 (61.1\%) fueron del sexo femenino y 93 (18.1\%) del sexo masculino.

Tabla 5. Estancia Hospitalaria de los pacientes sometidos a Colecistectomía Laparoscópica.

\begin{tabular}{lcccccc}
\hline Estancia Post Quirúrgica & \multicolumn{2}{c}{ Sexo } & \multicolumn{3}{c}{ Total } \\
& \multicolumn{2}{c}{ Femenino } & \multicolumn{2}{c}{ Masculino } & \\
& $\mathbf{N}^{\circ}$ & $\%$ & $\mathbf{N}^{\circ}$ & $\%$ & $\mathbf{N}^{\circ}$ & $\%$ \\
\hline$<=3$ días & 335 & 65.1 & 93 & 18.1 & 428 & 83.2 \\
\hline 4 - 8 días & 36 & 7.0 & 36 & 7.0 & 72 & 14.0 \\
\hline $9-13$ días & 3 & 0.6 & 7 & 1.4 & 10 & 2.0 \\
\hline $14-19$ días & 0 & & 1 & 0.2 & 1 & 0.2 \\
\hline $20-24$ días & 0 & & 2 & 0.4 & 2 & 0.4 \\
\hline $25-29$ días & 0 & & 1 & 0.2 & 1 & 0.2 \\
\hline TOTAL & 374 & 72.7 & 140 & 27.3 & 514 & 100 \\
\hline
\end{tabular}

Fuente: INICIB-FAMURP/ HOSPITAL DOS DE MAYO

\begin{tabular}{ll}
\hline Promedio & 2.18 días (01 - 12 días) \\
Desviación típica & 2,550 \\
$P$ & 0,001
\end{tabular}

La estancia hospitalaria en promedio fue de 2.18 días. Y fue menor o igual a 3 días en 428 pacientes (83.2\%), de los cuales 335 (65.1\%) fueron mujeres y 93 (18.1\%) varones. Se puede verificar que 4 pacientes $(0.8 \%)$ presentaron una estancia mayor de 14 días todos de sexo masculino. 


\section{DISCUSIÓN}

La colecistectomía laparoscópica es una de las intervenciones laparoscópicos más comúnmente realizados. Se usa pequeñas incisiones, provoca relativamente menos dolor, permite la deambulación precoz, requiere una estancia hospitalaria más corta, permite pronto regreso al trabajo y se asocia con una menor incidencia de una hernia incisional.

En nuestra investigación encontramos que el sexo femenino fue predominante: $73 \%$ versus $27 \%$ del sexo masculino, en una relación de 2.7:1; Giger $U$ et al encuentran una relación de 2:1 entre el sexo femenino y el masculino. Este predominio ha sido sobretodo relacionado a mujeres en edad reproductiva y multíparas, ya que con el pasar de los años la relación se va haciendo menor, por lo cual podemos inferir que el factor hormonal es el principal responsable de esta asociación $n^{4,5}$.

El promedio de edad fue de 45 años, el tiempo operatorio promedio de 86 minutos y la estancia hospitalaria en promedio fue 2.6 días similar a lo encontrado por Mohiuddin $\mathrm{K}$ et al, salvo en la estancia hospitalaria que fue de 1 día en promedio en dicho estudio, que es lo que también describen la mayoría de estudios; en nuestro medio la estancia hospitalaria es prolongada en relación a los estándares internacionales probablemente debido a factores sociales y administrativos del sistema de aseguramiento nacional ${ }^{6}$.

La estancia hospitalaria promedio fue de 2.18 días para el sexo femenino y 3.95 días para el sexo masculino; similar a lo encontrado por Rakan A et al quien reportó una estancia post operatoria mayor en hombres de $2.78 \pm 3.11$ días frente a $1.81 \pm 2.19$ días en mujeres ${ }^{7}$. Esto estaría asociado más a las complicaciones intraopertorias que a las post operatorias, aparentemente por la mayor dificultad que se tiene al identificar los elementos anatómicos en el género masculino, ya sea porque los varones llegan con vesículas más complicadas o con mayor tiempo de enfermedad, es así que diversos estudios como el de Mossa Ali et al, describen que el tiempo operatorio en el varón es mayor que en la mujer (81 vs 62 minutos) ${ }^{8}$. En nuestro estudio encontramos que es mayor el tiempo operatorio en el sexo masculino siendo 103 minutos en promedio versus 86 en promedio en el sexo femenino.

Dentro de las complicaciones intraoperatorias presentadas todas excepto la lesión del conducto cístico se presentaron con mayor frecuencia en el sexo masculino; siendo la más frecuente la disección compleja de los elementos anatómicos en el $4.5 \%$ frente a $3.7 \%$ del sexo femenino; esto es reportado por Bebko $S$ et al en su estudio como eventos intraoperatorios inesperados y que se presentan en el $50 \%$ de los casos, en coincidiendo con nuestros resultados que muestran a la disección compleja como la que se presenta con mayor frecuencia. Lo que estaría en relación a lo antes mencionado: la demora en el tratamiento quirúrgico, la presencia de una anatomía más distorsionada debido a una inflamación y en mayor medida a variantes anatómicas ${ }^{9}$.

La tasa de conversión a cirugía abierta también fue mayor en varones $3.1 \%$ frente a $0.8 \%$ en mujeres. Mossa Ali et al hallaron una tasa de conversión de $25 \%$ y $7 \%$ para el sexo masculino y femenino respectivamente, en nuestra serie la mayoría de conversiones se debieron a dificultad en la disección del triángulo de calot y anatomía poco clara por adherencias y en menor medida a hemorragia de difícil control y a otras complicaciones como perforación incidental de la vesícula biliar $^{8}$.

En relación a las complicaciones post operatorias la mayoría fue gradol. En el sexo masculino predominaron las complicaciones de grado II, grado IIla y IVa; las complicaciones encontradas fueron: anemia aguda con necesidad de transfusión, infecciones respiratorias como atelectasia que requirieron terapia antibiótica dentro de las más comunes. En las mujeres predominó el grado I, es decir emesis y dolor intraoperatorio intenso con mayor frecuencia, y el grado Illb, es decir hemoperitoneo con necesidad de laparotomía exploratoria por sangrado de lecho o de trócar; esto difiere con lo encontrado por Bocanegra Del Castillo $\mathrm{R}$ et al, quien reportó un total de 7 complicaciones en mujeres y 2 en varones la mayoría grado II. Es difícil encontrar la asociación de las complicaciones intraoperatorias con las post operatorias sino más bien en un manejo cuidadoso del post operatorio ${ }^{10}$.

Comodiagnóstico postoperatoriomásfrecuentemente encontrado tenemos el de colecistitis crónica litiásica para ambos sexos; sin embargo en el sexo masculino predominaron los diagnósticos de: piocolecisto, hidropiocolecisto y plastrón vesicular. Juárez $D$ et al describió que el diagnóstico de colecistitis crónica litiásica fue el más común en cirugías electivas; para cirugías de emergencia colecistitis aguda para mujeres y de hidrocolecisto para varones. Ambe P et reporta al sexo masculino como un factor de riesgo independiente para colecistitis grave ${ }^{11,12}$. 


\section{CONCLUSIÓN}

1. La estancia hospitalaria fue mayor en el sexo masculino que en el sexo femenino.

2. De julio del 2014 a julio del 2015 c de los pacientes colecistectomizados por vía laparoscópica en el Hospital Nacional el $73 \%$ fueron de sexo femenino y $27 \%$ del sexo masculino, con una relación de 2.7:1.

3. Los pacientes de sexo masculino con respecto al sexo femenino tienen mayor porcentaje de vesículas complicadas (piocolecisto, hidropiocolecisto y plastrón vesicular) en cirugías electivas, son cirugías con mayor tiempo operatorio por mayor porcentaje de complejidad en la disección, tienen mayor tasa de conversión a cirugía abierta, y 1 de cada 5 varones presentó algún grado de complicación.

4. La edad promedio de los pacientes fue de 45 años, con una edad relativamente mayor para el sexo masculino que para el femenino.

\section{Financiamiento: Autofinanciado.}

Conflicto de interés: Los autores declaran no tener conflictos de interés en la publicación de este artículo.

Recibido: 28 de febrero del 2017

Aprobado: 14 de marzo del 2017

\section{REFERENCIAS BIBLIOGRÁFICAS}

1. Yol S, Kartal A, Vatansev C, Aksoy F, Toy H. Sex as a factor in conversion from laparoscopic cholecystectomy to open surgery. JSLS. 2006;10(3):359-363.

2. Wiebke EA, Pruitt AL, Howard TJ, Jacobson LE, Broadie LE, Goulet RJ. Conversion of laparoscopic to open cholecystectomy: an analysis of risk factors. Surg Endosc. 1996; 10:742-745.

3. Al-Mulhim AA. Male gender is not a risk factor for the outcome of laparoscopic cholecystectomy: a single surgeon experience. Saudi J Gastroenterol. 2008;14:73-79.

4. Giger U, Michel J, Opitz I, Inderbitzin D, Kocher T, Krähenbühl L. Risk factors for perioperative complications in patients undergoing laparoscopic cholecystectomy: Analysis of 22,953 consecutive cases from the Swiss association of laparoscopic and thoracoscopic surgery database. Journal of the American College of Surgeons. 2006 Noviembre; 203(5): p. Pages 723-728.

5. Gaitán JA, Martínez VM. Enfermedad litiásica biliar, experiencia en una clínica de cuarto nivel del 2005-2011. Rev Colomb Cir. 2014; 29: p.188-96.

6. Mohiuddin K, Nizami S, Fitzgibbons RJ, Watson P, Memon B, Memon M. Predicting iatrogenic gall bladder perforation during laparoscopic cholecystectomy: a multivariate logistic regression analysis of risk factors. ANZ J Suergery. 2006; 76(3).
7. Rakan A, Wagih G, Mohammad A, Abdulrahman Q, Awdah A, Adel A. Role of male gender in laparoscopic cholecystectomy outcome. International Journal of Surgery and Medicine. 2015 Setiembre; 1(2): p. 38-42.

8. Moosa A, Idrees F, Sultan N, Ali S, Rao H, Mukhtiar A. Is male gender a tisk factor for cholecystectomy? Medical Channel. 2010 Octubre; 16(4): p. 541

9. Bebko S, Arrarte E, Larrabure L, Borda G, Samalvides F, Baracco V. Eventos intraoperatorios inesperados y conversión en pacientes colecistectomizados por vía laparoscópica: Sexo masculino como factor de riesgo independiente. Rev. Gastroenterol. Perú. 2011; 31(4): p. 335-344.

10. Bocanegra Del Castillo R, Córdova Cuadros M. Colecistectomía laparoscópica en el adulto mayor: complicaciones postoperatorias en mayores de 75 años en el Hospital Nacional Cayetano Heredia, Lima, Perú, del 2007 - 2011. Rev Gatroenterol Per. 2013; 33(2): p. 113-20.

11.Juárez D, Escamilla A, Miranda O, Hurtado J, Campos C, Canseco A. Género masculino factor de riesgo para la colecistectomía. Cirujano General. 2006 Enero; 28(2)

12. Ambe $P$, Weber $S$, Wassenberg $D$. Is gallbladder inflammation more severe in male patients presenting with acute cholecystitis? BMC Surgery. $2015 ; 15(48)$.

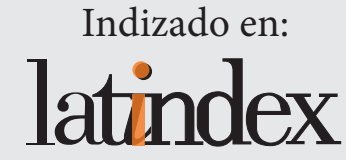

http://www.latindex.org/latindex/ficha?folio=14280

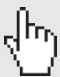

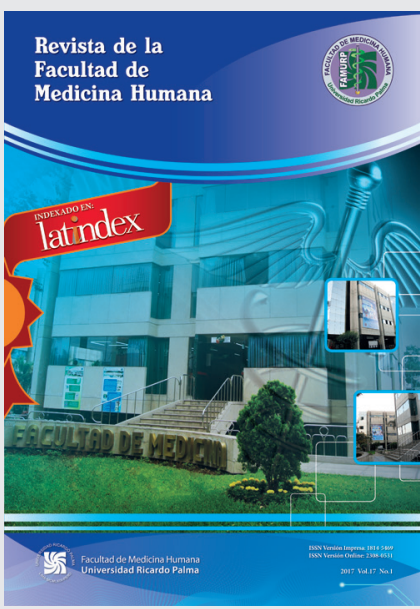

